\title{
Association of plasma D-dimer level with thromboembolic events after endovascular coil treatment of ruptured cerebral aneurysms
}

\author{
Hitoshi Fukuda, MD, ${ }^{1,2}$ Akira Handa, MD, ${ }^{2}$ Masaomi Koyanagi, MD, PhD, ${ }^{1,2}$ Benjamin Lo, MD, ${ }^{3}$ and \\ Sen Yamagata, MD ${ }^{1}$
}

Departments of ${ }^{1}$ Neurosurgery and ${ }^{2}$ Interventional Neuroradiology, Kurashiki Central Hospital, Kurashiki, Okayama, Japan; and ${ }^{3}$ Department of Neurosurgery, Montreal Neurological Institute and Hospital, McGill University Health Centre, Montreal, Quebec, Canada

OBJECTIVE Although endovascular therapy is favored for acutely ruptured intracranial aneurysms, hematological factors associated with acute subarachnoid hemorrhage $(\mathrm{SAH})$ may predispose to procedure-related ischemic complications. The aim of this study was to evaluate whether an elevated level of plasma D-dimer, a parameter of hypercoagulation in patients with acute $\mathrm{SAH}$, is correlated with increased incidence of thromboembolic events during endovascular coiling of ruptured aneurysms.

METHODS The authors analyzed data from 103 cases of acutely ruptured aneurysms (in 103 patients) treated with endovascular coil embolization at a single institution. Factors associated with elevated D-dimer level on admission were identified. The authors also evaluated whether D-dimer elevation was independently correlated with increased incidence of perioperative thromboembolic events.

RESULTS An elevated D-dimer concentration ( $\geq 1.0 \mu \mathrm{g} / \mathrm{ml})$ on admission was observed in $70(68.0 \%)$ of 103 patients. Increasing age ( $<<0.001$, Student t-test) and poor initial neurological grade representing World Federation of Neurosurgical Societies (WFNS) grade IV or V ( $p=0.0018$, chi-square test) were significantly associated with D-dimer elevation. Symptomatic thromboembolic events occurred in 11 cases (10.7\%). Elevated D-dimer levels on admission (OR 1.34, $95 \% \mathrm{Cl} 1.10-1.62, p=0.0029)$ independently carried a higher risk of thromboembolic events after adjustment for potential angiographic confounders, including wide neck of the aneurysm and large aneurysm size.

CONCLUSIONS Elevated D-dimer levels on admission of patients with acute SAH were significantly associated with increased incidence of thromboembolic events during endovascular coiling of ruptured aneurysms.

https://thejns.org/doi/abs/10.3171/2017.7.JNS171129

KEY WORDS D-dimer; endovascular coiling; intracranial aneurysm; thromboembolic event; subarachnoid hemorrhage; vascular disorders

$\mathrm{D}$ ESPITE advances in technology and techniques, thromboembolic events are still encountered as inherent perioperative complications of endovascular coil embolization of cerebral aneurysms. ${ }^{17,23}$ Anatomical factors, including wide aneurysm neck, small parent artery diameter, and branches arising from aneurysmal walls, were reported as risk factors for thromboembolic events. ${ }^{6,21}$

Procedure-related thromboembolic events are observed more frequently in endovascular coiling of ruptured aneu- rysms than in coiling of unruptured ones. ${ }^{17}$ In the treatment of ruptured aneurysms, hypercoagulability as a systemic response to acute subarachnoid hemorrhage $(\mathrm{SAH})^{9,13}$ may be associated with an increased incidence of thromboembolic events in addition to the aforementioned anatomical factors. Among possible biomarkers of hypercoagulability, $\mathrm{D}$-dimer concentration is known to be elevated in the acute stages of $\mathrm{SAH}^{9,10,13}$ and is a useful marker to detect sources of thromboembolism in cerebral ischemic strokes. ${ }^{16}$ In this study, we investigate whether levels of D-dimer, a stable

ABBREVIATIONS IQR = interquartile range; $\mathrm{SAH}=$ subarachnoid hemorrhage; WFNS = World Federation of Neurosurgical Societies .

SUBMITTED May 5, 2017. ACCEPTED July 24, 2017.

INCLUDE WHEN CITING Published online February 9, 2018; DOI: 10.3171/2017.7.JNS171129. 
parameter of hypercoagulability and coagulation cascade turnover, ${ }^{1,12}$ are associated with an increased incidence of symptomatic thromboembolic events after endovascular therapy for aneurysmal SAH.

\section{Methods}

The study is reported based on criteria from the Strengthening the Reporting of Observational Study in Epidemiology (STROBE) statement. ${ }^{24}$ The study protocol was approved by the Kurashiki Central Hospital Research Ethics Committee, and waiver of consent was sought and obtained for this cohort study with no unique patient identifiers.

\section{Patient Selection and Study Design}

This is a retrospective cohort study including patients who underwent endovascular coil embolization of ruptured cerebral aneurysms within 72 hours of symptom onset in our institution between January 2012 and January 2017. The period of 72 hours was determined to exclude patients with delayed cerebral ischemia due to cerebral vasospasm. In fact, the majority $(85 \%)$ of our patients were treated within 24 hours of symptom onset. Our study also included patients who underwent coil embolization between 24 and 72 hours after symptom onset in order to capture those who had a good initial World Federation of Neurosurgical Societies (WFNS) ${ }^{5}$ grade and whose hospital visit was delayed because their symptoms were less severe, as well as those who had a poor initial WFNS grade but demonstrated clinical improvement after treatment of concomitant processes, such as hydrocephalus, seizure, and intracranial pressure elevation. In order to properly detect procedure-related symptomatic thromboembolic events after aneurysmal embolization, we excluded patients who underwent endovascular parent artery occlusion $(n=9)$ and those who experienced intraprocedural aneurysmal rupture $(n=4)$. Patients with initial severe neurological status, representing WFNS grade V, were also excluded when they were persistently comatose more than 48 hours after embolization $(n=13)$, because they were not at risk for new neurological deficits. In patients with multiple aneurysms, only the single culprit aneurysm that was most likely to have ruptured was determined by clot distribution and aneurysmal morphology. As a result, medical and surgical records of 103 consecutive patients with 103 treated aneurysms were retrieved and reviewed.

The choice of treatment-surgical clipping or endovascular coil embolization-was determined according to multidisciplinary discussions involving both surgical and endovascular teams. In general, surgical clipping was preferred for younger patients with less comorbid burden, very small aneurysmal sizes, or aneurysms with wide necks or aberrant branches. Microsurgery was also indicated for patients requiring hematoma evacuation and decompressive craniectomy. Endovascular coiling was selected for older patients with comorbidities, posterior circulation aneurysms, or small-necked aneurysms.

Thromboembolic events as a primary outcome in this study were defined as the occurrence of a new focal neurological deficit within 48 hours of the endovascular coiling, with corresponding areas of hypodensity on head CT. Diffusion-weighted MRI was obtained when CT findings did not account for neurological deficits. Whether neurological deficits corresponded to CT or MRI lesions was determined by a physician (S.Y.) who was blinded to patients' admission D-dimer levels. Neither asymptomatic lesions nor neurological deficits secondary to other causes (such as hematoma formation and brain swelling) were regarded as thromboembolic events.

Patients' baseline characteristics, initial head CT findings, and initial neurological status represented by WFNS grade were included to test for any association of these variables with D-dimer elevation on admission. Subsequently, any correlation between D-dimer level and thromboembolic events was investigated by means of univariate analysis. Finally, other independent variables were also investigated for risk of thromboembolic events, including aneurysmal morphology by angiography, type of anesthesia (general or local), use of a balloon-assisted technique, and patients' baseline characteristics, and then multivariable analysis was performed.

\section{Radiological Evaluation}

All patients underwent a head CT scan on admission. The subarachnoid clot burden on admission CT was classified by the modified Fisher scale, taking into account the presence of intraventricular hemorrhage and intracerebral hematoma ${ }^{8}$ Neck width, aneurysm height (distance from the neck center to the top of the dome), aneurysm width (perpendicular to the aneurysm height), and adjacent parent artery diameter were measured on a $0.1-\mathrm{mm}$ scale by $3 \mathrm{D}$ rotational angiography. The maximum measurement of aneurysm height or aneurysm width was defined as the aneurysm size. Aneurysm size $>10 \mathrm{~mm}$ was defined as large. Aneurysms with a neck width $>4 \mathrm{~mm}$ were classified as wide necked. Parent artery diameter $<1.5 \mathrm{~mm}$ was defined as small parent artery diameter. ${ }^{4,6,18}$

\section{D-Dimer Measurement}

Because D-dimer is useful in estimating thromboembolic sources of cerebral ischemic strokes, our emergency stroke department has incorporated D-dimer level as part of the routine admission blood work for all stroke types, including both ischemic and hemorrhagic subtypes, since 2012. Blood samples were obtained from all 103 patients within 1 hour of admission. Free-flowing blood was collected into polyethylene terephthalate tubes containing sodium citrate $(3.2 \%, 0.11 \mathrm{~mol} / \mathrm{L})$ as the anticoagulant at a ratio of 1 volume to 9 volumes of blood. The samples were centrifuged for 10 minutes at $3000 \mathrm{rpm}$ (at $22^{\circ} \mathrm{C}$ ) to separate the plasma. D-dimer was measured with a quantitative photometric latex immunoassay (Tinaquant; Roche Diagnostics $\mathrm{GmbH}$ ). According to the manufacturer's instructions and the institutional agreement, a value of D-dimer $\geq 1.0 \mu \mathrm{g} / \mathrm{ml}$ was defined as elevated.

\section{Endovascular Procedure}

Coil embolization was performed in an angiography suite (Allura Xper FD20/20; Philips Healthcare) under either general or local anesthesia. General anesthesia was 
preferred to ensure immobilization and adequate hemodynamic control, whereas local anesthesia was generally restricted to limited patients with severe comorbidities. All endovascular procedures were performed under systemic heparinization. An intravenous bolus dose of 4000 or 5000 units of heparin was given immediately after the sheath introducer was inserted. Activated clotting time was measured every 30 minutes, and an additional bolus dose of 1000 or 2000 units of heparin was administered for a goal of an activated clotting time of between 250 and 300 seconds. Postprocedurally, heparin was not reversed with protamine in any case. A bolus dose of oral antiplatelet agent was not administered for embolization in any case prior to the procedure. An Excelsior SL-10 microcatheter (Stryker Neurovascular) was navigated into the aneurysm with the aid of a CHIKAI microguidewire (ASAHI INTECC), through a 6-Fr or 7-Fr guiding catheter (Roadmaster; Goodman Co. Ltd.) inserted in the femoral or brachial artery. Subsequently, detachable platinum coils, such as Target (Stryker Neurovascular), Trufill DCS Orbit (Codman and Shurtleff, Inc. and Johnson \& Johnson), and ED (Kaneka Medics Corp.), were placed into the aneurysm. All lines were continuously irrigated with pressurized heparinized saline during the procedure, and the microcatheter was manually flushed after placement of each platinum coil. A balloon-assisted technique, with a compliant balloon catheter (Sceptor; MicroVention, Inc.), was used if possible. A balloon was inflated to prevent extrusion of inserted coils or microcatheters from the aneurysm so that each inflation time did not exceed 2 minutes. Stent-assisted coil embolization was not performed. When major vessels represented severe stenosis or occlusion by thrombus formation during the procedure, heparin was added to raise the activated clotting time to around 300 seconds, sodium ozagrel $(80 \mathrm{mg})$ was intravenously administered, and mechanical thrombectomy using a microcatheter was attempted. Thrombolysis using urokinase or tissue plasminogen activator was not performed. Glycoprotein IIb/IIIa inhibitors, such as eptifibatide (Integrilin) or abciximab (ReoPro), were not used as such agents are not yet approved in Japan. The occlusion grade after endovascular treatment was classified as complete occlusion, neck remnant, or residual aneurysm by using a modified Raymond scale. ${ }^{19}$ We were not necessarily able to retrieve the procedure duration time from the patients' records, but it was between 1.5 and 3 hours in most cases.

\section{Data Analysis}

Quantitative variables are expressed as the mean \pm standard deviation or the median value and interquartile range (IQR), as appropriate. Chi-square analysis was used to test associations between categorical variables. Normality of the data was evaluated using the Shapiro-Wilk test. Normally distributed continuous variables were analyzed by Student t-test. Nonnormally distributed continuous variables were analyzed by Mann-Whitney U-test. The risk of thromboembolic events associated with D-dimer level was evaluated by univariate logistic regression. Univariate logistic regression analysis was also performed to detect significant associations of thromboembolic events with other covariates. Only variables with $\mathrm{p}<0.10$ in the univariate
TABLE 1. Characteristics of 103 patients who underwent endovascular coil embolization of ruptured aneurysms

\begin{tabular}{lc}
\hline \multicolumn{1}{c}{ Variable } & Value \\
\hline Mean age in yrs & $65.9 \pm 16.2$ \\
\hline Female sex & $89(86.4)$ \\
\hline Hypertension & $54(52.4)$ \\
\hline Type 2 diabetes & $5(4.9)$ \\
\hline Hyperlipidemia & $18(17.5)$ \\
\hline Antiplatelet/anticoagulant use & $7(6.8)$ \\
\hline WFNS grade & $37(35.9)$ \\
\hline I & $36(35.0)$ \\
\hline II & $1(1.0)$ \\
\hline III & $18(17.5)$ \\
\hline IV & $11(10.7)$ \\
\hline V & $54(52.4)$ \\
\hline Modified Fisher scale grade of 3 or 4 & 1.6 \\
\hline D-dimer level, $\mu g / m l$ & $0.8-3.25$ \\
\hline Median
\end{tabular}

Data are presented as number of patients (\%) unless otherwise indicated. Means are presented with SDs.

analysis were included in the multivariable logistic regression model-building process. Models were built using forward/backward stepwise logistic regression with variables entered into the model and removed at a 0.10 significance level. Odds ratios and 95\% confidence intervals were also determined in the logistic regression analysis. Commercially available software (IBM SPSS version 20; IBM Corp.) was used for all statistical analyses.

\section{Results}

\section{Baseline Characteristics of Patients and Treated Aneurysms}

Patient baseline characteristics are summarized in Table 1. According to our endovascular database, 103 patients (14 men and 89 women) with a mean age of $65.9 \pm 16.2$ years underwent endovascular coiling within 72 hours of symptom onset. Of these 103 patients, 54 (52.4\%) had a medical history of hypertension, $5(4.9 \%)$ had type 2 diabetes, 18 $(17.5 \%)$ had hyperlipidemia, and 7 (6.8\%) had taken oral antiplatelet or anticoagulation agents. Eleven patients who presented with initial WFNS grade V were included because they responded to conservative treatment or external ventricular drainage before or within 48 hours of coiling, and thus we were able to evaluate focal neurological deficits. Thick subarachnoid clot classified as modified Fisher scale grade 3 or 4 was observed in 54 patients (52.6\%).

Characteristics of the aneurysms and a summary of endovascular coiling are presented in Table 2 . In terms of aneurysm morphology, the mean aneurysm size was 6.6 $\pm 3.4 \mathrm{~mm}$, and 17 aneurysms (16.5\%) were larger than 10 $\mathrm{mm}$ in height or width. The mean neck width was $3.6 \pm 1.8$ $\mathrm{mm}$, and 30 aneurysms $(29.1 \%)$ had a wide neck ( $>4 \mathrm{~mm})$. The diameter of the parent artery was $<1.5 \mathrm{~mm}$ in 21 
TABLE 2. Characteristics of 103 aneurysms and summary of endovascular coiling

\begin{tabular}{lc}
\hline \multicolumn{1}{c}{ Variable } & Value \\
\hline Anterior circulation & $78(75.7)$ \\
\hline Aneurysm size & $6.6 \pm 3.4$ \\
\hline Mean size in mm & $17(16.5)$ \\
\hline No. of large aneurysms $(>10 \mathrm{~mm})$ & \\
\hline Neck width & $3.6 \pm 1.8$ \\
\hline Mean width (mm) & $30(29.1)$ \\
\hline No. of aneurysms w/ wide neck $(>4 \mathrm{~mm})$ & $21(20.4)$ \\
\hline Parent artery diameter <1.5 mm & $9(8.7)$ \\
\hline Local anesthesia & $61(59.2)$ \\
\hline Balloon-assisted technique & \\
\hline Aneurysm occlusion grade & $45(43.7)$ \\
\hline Complete occlusion & $47(45.6)$ \\
\hline Neck remnant & $11(10.7)$ \\
\hline Residual aneurysm & $6(5.8)$ \\
\hline Intraoperative thrombus formation & $11(10.7)$ \\
\hline Thromboembolic events
\end{tabular}

Data are presented as number of patients or aneurysms (\%) unless otherwise indicated. Means are presented with SDs.

cases (20.5\%); in most of these cases, the aneurysms were located on peripheral arteries, including anterior cerebral, posterior cerebral, and posterior inferior cerebellar arteries. In 9 cases $(8.7 \%)$, the aneurysms were treated under local anesthesia, because of the patients' general condition and comorbid burden. Balloon assistance, with placement of a compliant balloon catheter at the orifice of the aneurysm, was used in 61 (59.2\%) cases. Aneurysm dome occlusion was achieved (complete occlusion or neck remnant based on the modified Raymond scale) in 92 aneurysms (89.3\%). Intraoperative thrombus formation in the parent artery was observed in 6 cases (5.8\%), and symptomatic thromboembolic events occurred in 11 cases (10.7\%).

\section{Risk Factors for D-Dimer-Level Elevation on Admission}

An elevated D-dimer level ( $\geq 1.0 \mu \mathrm{g} / \mathrm{ml}$ ) on admission was observed in $70(68.0 \%)$ of 103 patients. Table 3 shows the results of univariate analysis of risk factors for Ddimer-level elevation. Increasing age $(\mathrm{p}<0.001$, Student t-test) and female sex $(p=0.013$, chi-square test) were significantly associated with D-dimer-level elevation. Poorer initial neurological status (WFNS grade IV or V) was also significantly correlated with D-dimer-level elevation ( $\mathrm{p}=$ 0.018 ) by chi-square test. The admission D-dimer level was elevated in all 7 of the patients who had taken oral antiplatelet or anticoagulation agents, although the difference was not statistically significant.

\section{Relationship Between D-Dimer and Thromboembolic Events}

Thrombus formation in the parent artery during endovascular coiling was observed in 6 patients (5.8\%). An increasing level of D-dimer was significantly associated
TABLE 3. Univariate analysis of factors for increased plasma D-dimer level

\begin{tabular}{lccc}
\hline \multirow{2}{*}{ Variable } & \multicolumn{2}{c}{ D-Dimer Level } & \\
\cline { 2 - 3 } & $\begin{array}{c}\text { Elevated } \\
(\mathrm{n}=70)\end{array}$ & $\begin{array}{c}\text { Normal } \\
(\mathrm{n}=33)\end{array}$ & $\begin{array}{c}\mathrm{p} \\
\text { Value }\end{array}$ \\
\hline Mean age in yrs & $70.5 \pm 15.2$ & $56.1 \pm 14.0$ & $<0.001^{*}$ \\
\hline Female sex & $65(92.9)$ & $24(72.7)$ & $0.013^{*}$ \\
\hline Hypertension & $35(50.0)$ & $19(57.6)$ & 0.61 \\
\hline Type 2 diabetes & $3(4.3)$ & $2(6.1)$ & 0.65 \\
\hline Hyperlipidemia & $13(18.6)$ & $5(15.2)$ & 0.88 \\
\hline Antiplatelet/anticoagulant & $7(10.0)$ & $0(0)$ & 0.09 \\
\hline WFNS grade IV or V & $25(35.7)$ & $4(12.1)$ & $0.018^{*}$ \\
\hline Modified Fisher scale grade 3 or 4 & $40(57.7)$ & $14(42.4)$ & 0.24 \\
\hline
\end{tabular}

Data are presented as number of patients (\%) unless otherwise indicated. Means are presented with SDs. The $p$ value for mean age is based on the Student $t$-test; all other $p$ values are based on chi-square tests.

* Statistically significant on univariate analysis $(p<0.05)$.

with an increased incidence of thrombus formation in univariate analysis ( $p=0.015$, chi-square test). None of the anatomical factors, including a wide aneurysm neck, large aneurysm size, and small diameter of the parent artery, were revealed as a significant risk factor for thrombus formation. Of 6 patients with intraoperative thrombus formation, 3 (50\%) suffered symptomatic thromboembolic events. Intraoperative thrombus formation was a significant risk factor for symptomatic thromboembolic events ( $p=0.015$, Mann-Whitney U-test).

Symptomatic thromboembolic events occurred in 11 cases (10.7\%), with cerebral infarctions associated with neurological deficits evident in the cortical region (7 cases), in the perforator region (3 cases), and in both the cortical and perforator regions (1 case) on the postoperative imaging. Thromboembolic events occurred during embolization of internal carotid artery (6 cases), anterior communicating artery ( 2 cases), basilar artery (1 case), vertebral artery (1 case), and posterior cerebral artery (1 case) aneurysms. Table 4 shows the results of univariate analysis of risk factors for thromboembolic events. Plasma D-dimer level was significantly correlated with increased incidence of thromboembolic events (OR 1.36, 95\% CI $1.13-1.63, p=0.0011)$. In terms of aneurysm morphology, wide neck ( $>4 \mathrm{~mm}$, OR $5.25,95 \%$ CI $1.41-19.6, \mathrm{p}=0.014)$ was also associated with a risk of thromboembolic events. Association with large aneurysm size (>10 mm, OR 3.43 , $95 \%$ CI $0.88-13.4, p=0.076$ ) was marginally significant. Two representative cases of thromboembolic events are shown in Figs. 1 and 2, where either an elevated D-dimer level or a wide neck was relevant as a risk factor.

On multivariable logistic regression analysis, D-dimer level (OR 1.34, 95\% CI 1.10-1.62, p = 0.0029) remained a significant risk factor for thromboembolic events after adjustment for potential confounders, including wide neck $(>4 \mathrm{~mm})$ and large aneurysm size (> $10 \mathrm{~mm})$ (Table 5).

\section{Discussion}

In this article, we demonstrated that plasma D-dimer 
TABLE 4. Univariate logistic regression analyses of risk factors for thromboembolic events

\begin{tabular}{lcccc}
\hline \multicolumn{1}{c}{ Variable } & TEE $(\mathrm{n}=11)$ & No TEE $(\mathrm{n}=92)$ & OR $(95 \% \mathrm{Cl})$ & $\mathrm{p} \mathrm{Value}$ \\
\hline Mean age in yrs & $69.5 \pm 16.1$ & $65.5 \pm 16.3$ & $1.02(0.98-1.06)$ & 0.45 \\
\hline Female sex & $10(90.9)$ & $79(85.9)$ & $1.65(0.19-13.9)$ & 0.65 \\
\hline Hypertension & $7(63.6)$ & $47(51.1)$ & $1.68(0.46-6.10)$ & 0.44 \\
\hline Type 2 diabetes & $1(9.1)$ & $4(4.3)$ & $2.20(0.22-21.6)$ & 0.50 \\
\hline Hyperlipidemia & $1(9.1)$ & $14(15.2)$ & $0.66(0.07-5.89)$ & 0.71 \\
\hline Antiplatelet/anticoagulant & $1(9.1)$ & $6(6.5)$ & $1.43(0.16-13.1)$ & 0.75 \\
\hline Anterior circulation & $8(72.7)$ & $70(76.1)$ & $0.84(0.20-3.44)$ & 0.81 \\
\hline Large aneurysm $(>10 \mathrm{~mm})$ & $4(36.4)$ & $13(14.1)$ & $3.43(0.88-13.4)$ & $0.076 \dagger$ \\
\hline Wide neck $>4$ mm) & $7(63.6)$ & $23(25.0)$ & $5.25(1.41-19.6)$ & $0.014^{*}$ \\
\hline Parent artery<1.5 mm & $2(18.2)$ & $19(20.7)$ & $0.85(0.17-4.29)$ & 0.85 \\
\hline Local anesthesia $(\%)$ & $2(18.2)$ & $7(7.6)$ & $2.67(0.48-14.8)$ & 0.26 \\
\hline Balloon-assisted technique & $6(54.5)$ & $55(59.8)$ & $0.81(0.23-2.84)$ & 0.74 \\
\hline Residual aneurysm & $3(27.2)$ & $8(8.7)$ & $3.07(0.70-13.5)$ & 0.14 \\
\hline D-dimer $(\mu g / m l)$ & & & 1.36 & $0.0011^{*}$ \\
\hline Median & 4.5 & 1.5 & $1.13-1.63$ & \\
\hline IQR & $3.3-8.95$ & $0.7-2.725$ & & \\
\hline
\end{tabular}

TEE = thromboembolic event.

Data are presented as number of patients or aneurysms (\%) unless otherwise indicated. Means are presented with SDs.

* Statistically significant difference on univariate analysis $(p<0.05)$.

$\dagger$ Marginally significant difference on univariate analysis $(p<0.10)$.

level on admission was significantly associated with increased incidence of thromboembolic events during endovascular coiling of ruptured cerebral aneurysms.

Endovascular coil embolization is favored for treatment of ruptured cerebral aneurysms because it is less invasive than surgical clip placement. When both surgical clipping and endovascular coiling are therapeutic options for ruptured aneurysms, the outcome of endovascular coiling is superior to that of surgical clipping. ${ }^{15}$ However, endovascular coiling involves insertion of coaxial catheters into the parent artery and detachment of platinum coils as foreign bodies in the aneurysm, thus predisposing to thromboembolic events. Procedure-related thromboembolic events are observed more frequently in endovascular coiling of ruptured aneurysms than in coiling of unruptured ones. ${ }^{16}$ Several possible reasons may explain the increased propensity of thromboembolic events in the treatment of ruptured aneurysms. First, even in elderly patients, ruptured aneurysms must be treated, whereas unruptured aneurysms can be kept untreated and monitored. Catheterization into tortuous parent arteries with atherosclerosis increases the likelihood of thromboembolic events in elderly patients. Second, although some patients with ruptured aneurysms may be taking antiplatelet medication for comorbid conditions, pretreatment with antiplatelet medication is not performed in these cases for the purpose of preventing thromboembolic events associated with endovascular coiling. In contrast, patients undergoing endovas-
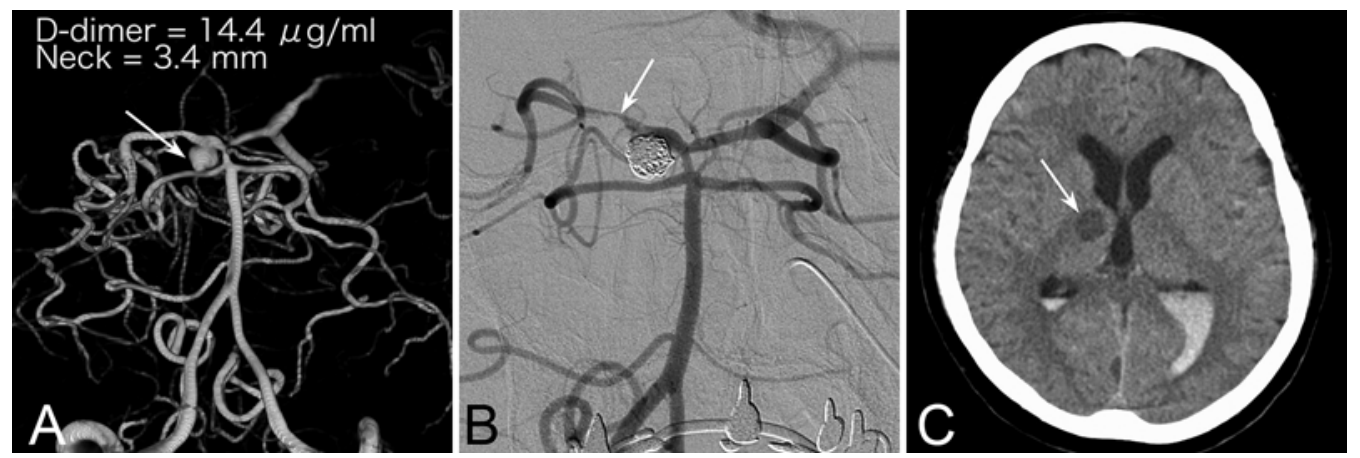

FIG. 1. Images obtained in a 58-year-old woman who presented with WFNS grade I SAH. Her plasma D-dimer level on admission was $14.4 \mu \mathrm{g} / \mathrm{ml}$. A: 3D-rotational right vertebral artery (VA) angiogram showing a ruptured right posterior cerebral artery (PCA) aneurysm (arrow). The neck of the aneurysm was measured as $3.4 \mathrm{~mm}$. B: Anteroposterior view of right VA angiogram obtained at the end of the coil embolization procedure. Intraluminal thrombus formation is depicted as a contrast filling defect of the $\mathrm{P}_{2}$ portion of the right PCA (arrow). C: Postoperative head CT scan showing perforator region infarction in the right thalamus (arrow). 

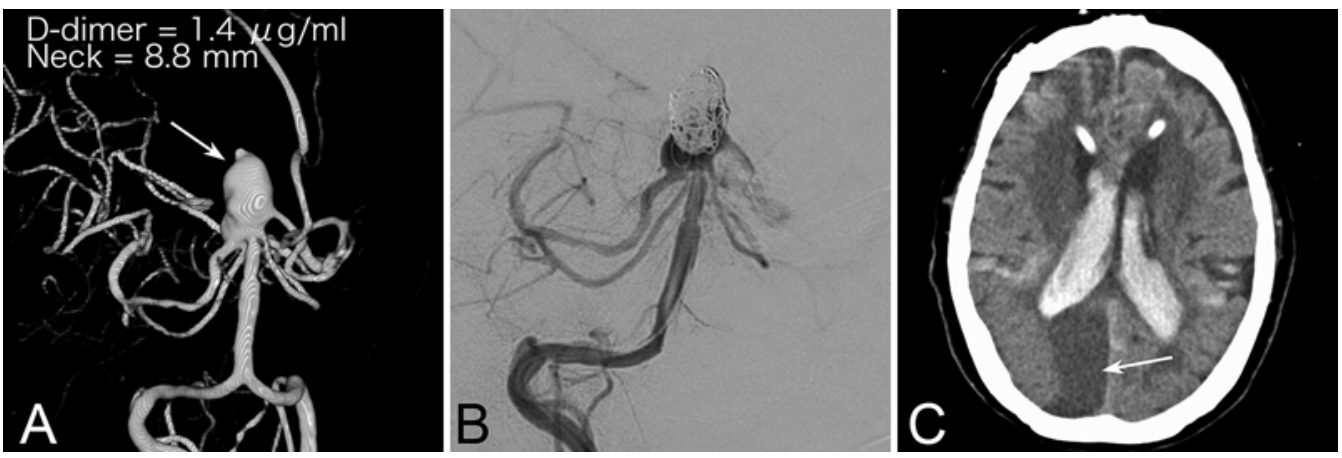

FIG. 2. Images obtained in a 69-year-old woman who presented with WFNS grade IV SAH. Her plasma D-dimer level on admission was $1.4 \mu \mathrm{g} / \mathrm{ml}$. A: 3D-rotational right VA angiogram showing a ruptured basilar artery bifurcation aneurysm (arrow). The neck of the aneurysm was measured as $8.8 \mathrm{~mm}$. B: Anteroposterior view of right VA angiogram obtained at the end of coil embolization. Residual aneurysm is observed at the bottom part of the lesion. C: Postoperative head CT scan showing cortical region infarction at the right occipital lobe (arrow).

cular coiling of unruptured aneurysms are commonly prescribed single- or dual-antiplatelet therapy as a pretreatment to prevent thromboembolic events. ${ }^{11,26}$ In addition, hypercoagulopathic states associated with acute $\mathrm{SAH}^{9,13}$ may trigger and exacerbate thrombus formation during and after treatment. Because D-dimer correlates well with other hemostatic parameters and represents upregulated hemostasis in aneurysmal SAH, we hypothesized that an elevated D-dimer level may predict increased incidence of thromboembolic events related to endovascular coiling of ruptured cerebral aneurysms.

In endovascular coiling of cerebral aneurysms, thrombus formation is often encountered at the interface of the coil and luminal blood flow and has been reported as a primary risk factor for symptomatic thromboembolic events. ${ }^{25,27}$ Thus, a wide aneurysm neck was reported to be associated with an increased incidence of thromboembolic events due to a larger coil-lumen interface in the treatment of unruptured aneurysms..$^{6,21}$ In addition, our results suggest that hypercoagulability associated with $\mathrm{SAH}$ also contributes to thrombus formation and the incidence of thromboembolic events in the treatment of ruptured aneurysms. On the other hand, 8 of 11 cases of thromboembolic events were not associated with thrombus formation around the aneurysm neck, suggesting other sources of thromboembolism. Although the rescue therapy protocol described in Methods may have biased the incidence of thromboembolic events in the subgroup of patients with observed intraoperative thrombus formation, one possible explanation of thromboembolic events without thrombus formation around the aneurysm neck is thrombus formed through shear stress between catheters and parent vessels with atherosclerosis. ${ }^{6}$ The plasma level of D-dimer may be elevated not only as an acute systemic response for SAH but also depending on patients' premorbid status, including aging. ${ }^{10,20}$ Atherosclerotic change is presumed to be one of the causes of D-dimer elevation in elderly patients. ${ }^{10,14}$ Thromboembolic events without visible thrombus formation at the neck of the aneurysm during endovascular coiling may be caused by catheter insertion into a diseased parent vessel, as represented by elevated D-dimer levels. ${ }^{6}$ In addition, chronic derangements in systemic coagulation states from comorbid burden, such as deep venous thrombosis and malignancies, may be associated with D-dimer elevations and thromboembolic events unrelated to endovascular procedures, ${ }^{10,22}$ although screening for these pathologies was not performed in the present study.

It is well known that antiplatelet therapy reduces thromboembolic events in endovascular coiling of unruptured aneurysms. ${ }^{11,26}$ Likewise, preoperative loading with an antiplatelet agent may be helpful to prevent thromboembolic events in the treatment of ruptured cerebral aneurysms. However, because aneurysmal SAH is associated with hemorrhagic complications, including clot-induced pial injury and external ventricular drainage-related hemorrhage, routine use of antiplatelet agents in the treatment of ruptured aneurysms may not be justified. ${ }^{2,6,7}$ Elective use of intraprocedural aspirin at a dose of $650 \mathrm{mg}$ for patients with angiographic high-risk features was reported to reduce thromboembolic events in the treatment of ruptured aneurysms without significantly increasing hemorrhagic complications. ${ }^{6}$ A similar approach may be beneficial to reduce thromboembolic complications in patients with elevated D-dimer levels. When activated by stagnant arterial flow or intimal injury during endovascular coiling, platelets aggregate, creating a scaffold for coagulation factors to form coagulation thrombi. ${ }^{3}$ Although D-dimer elevation represents hypercoagulation, antiplatelet therapy may contribute to the reduction of thromboembolic events by blocking the initial step of this cascadic platelet-coagulation interaction. In addition, antiplatelet therapy may also reduce shear stress between endovascular devices and diseased parent vessels in patients with atherosclerosis.

The limitations of this study include its retrospective design and the fact that it involved only a single institution. Selection bias is present in that coiling is preferred for elderly patients. Inclusion of more elderly patients, who had elevated D-dimer levels and atherosclerotic burden, may have caused increased incidence of thromboembolic events, although the rate of thromboembolic events in our study $(10.7 \%)$ was comparable to rates in previous studies $(5.5 \%-11 \%) .^{17,23}$ Exclusion of asymptomatic CT or MRI lesions, as in previous studies, ${ }^{17,23}$ may have led to underestimation of the actual incidence of thrombo- 
TABLE 5. Multivariable logistic regression analysis of risk factors for thromboembolic events $\dagger$

\begin{tabular}{lcl}
\hline \multicolumn{1}{c}{ Variable } & Adjusted OR $(95 \% \mathrm{Cl})$ & p Value \\
\hline D-dimer level in $\mu \mathrm{g} / \mathrm{ml}$ & $1.34(1.10-1.62)$ & $0.0029^{*}$ \\
\hline Wide neck $(>4 \mathrm{~mm})$ & $3.90(0.90-16.8)$ & 0.068 \\
\hline
\end{tabular}

* Statistically significant difference on multivariable analysis $(p<0.05)$.

$\dagger$ Adjusted for large aneurysm (> $10 \mathrm{~mm}$ ).

embolic phenomena. In this study, we excluded patients with poor-grade SAH presenting in a persistent coma because of the inability to detect new neurological deficits after treatment. This patient subgroup may have distinct pathophysiological mechanisms in coagulopathy, D-dimer elevation, and procedure-related thromboembolic events. In addition, procedure duration, which may be a potential risk factor for thromboembolic events, was not included as an independent variable. Finally, association of elevated D-dimer levels with increased incidence of venous thrombosis (e.g., deep venous thrombosis and pulmonary embolism) remains to be elucidated. In this article, we would like to emphasize that SAH patients with elevated D-dimer levels on admission were at higher risk of procedurerelated thromboembolic events when treated with endovascular coiling. Elective use of antiplatelet agents might help to prevent thromboembolic events in this subgroup, and surgical clipping may be indicated unless surgery is critically challenging. Further investigation is warranted to address the optimal treatment of ruptured aneurysms in patients with elevated D-dimer levels, as well as causative mechanisms involved in procedure-related thromboembolic events.

\section{Conclusions}

A stable parameter of a hypercoagulation state in patients with acute SAH, an elevated D-dimer level on admission was significantly associated with an increased incidence of thromboembolic events during endovascular coiling of ruptured aneurysms.

\section{References}

1. Barber M, Langhorne P, Rumley A, Lowe GD, Stott DJ: Hemostatic function and progressing ischemic stroke: D-dimer predicts early clinical progression. Stroke 35:14211425, 2004

2. Bodily KD, Cloft HJ, Lanzino G, Fiorella DJ, White PM, Kallmes DF: Stent-assisted coiling in acutely ruptured intracranial aneurysms: a qualitative, systematic review of the literature. AJNR Am J Neuroradiol 32:1232-1236, 2011

3. Cosemans JM, Angelillo-Scherrer A, Mattheij NJ, Heemskerk JW: The effects of arterial flow on platelet activation, thrombus growth, and stabilization. Cardiovasc Res 99:342-352, 2013

4. Derdeyn CP, Cross DT III, Moran CJ, Brown GW, Pilgram TK, Diringer MN, et al: Postprocedure ischemic events after treatment of intracranial aneurysms with Guglielmi detachable coils. J Neurosurg 96:837-843, 2002

5. Drake CG: Report of World Federation of Neurological Surgeons Committee on a Universal Subarachnoid Hemorrhage Grading Scale. J Neurosurg 68:985-986, 1988 (Letter)
6. Edwards NJ, Jones WH, Sanzgiri A, Corona J, Dannenbaum M, Chen PR: Antiplatelet therapy for the prevention of pericoiling thromboembolism in high-risk patients with ruptured intracranial aneurysms. J Neurosurg 127:1326-1332, 2017

7. Egashira Y, Yoshimura S, Enomoto Y, Ishiguro M, Asano T, Iwama T: Ultra-early endovascular embolization of ruptured cerebral aneurysm and the increased risk of hematoma growth unrelated to aneurysmal rebleeding. J Neurosurg 118:1003-1008, 2013

8. Frontera JA, Claassen J, Schmidt JM, Wartenberg KE, Temes R, Connolly ES Jr, et al: Prediction of symptomatic vasospasm after subarachnoid hemorrhage: the modified fisher scale. Neurosurgery 59:21-27, 2006

9. Fujii Y, Takeuchi S, Sasaki O, Minakawa T, Koike T, Tanaka R: Hemostasis in spontaneous subarachnoid hemorrhage. Neurosurgery 37:226-234, 1995

10. Fukuda H, Lo B, Yamamoto Y, Handa A, Yamamoto Y, Kurosaki Y, et al: Plasma D-dimer may predict poor functional outcomes through systemic complications after aneurysmal subarachnoid hemorrhage. J Neurosurg 127:284-290, 2017

11. Hwang G, Jung C, Park SQ, Kang HS, Lee SH, Oh CW, et al: Thromboembolic complications of elective coil embolization of unruptured aneurysms: the effect of oral antiplatelet preparation on periprocedural thromboembolic complication. Neurosurgery 67:743-748, 2010

12. Ilveskero S, Juvela S, Siironen J, Lassila R: D-dimer predicts outcome after aneurysmal subarachnoid hemorrhage: no effect of thromboprophylaxis on coagulation activity. Neurosurgery 57:16-24, 2005

13. Juvela S, Siironen J: D-dimer as an independent predictor for poor outcome after aneurysmal subarachnoid hemorrhage. Stroke 37:1451-1456, 2006

14. Kario K, Matsuo T, Kobayashi H: Which factors affect high D-dimer levels in the elderly? Thromb Res 62:501-508, 1991

15. Molyneux AJ, Kerr RS, Yu LM, Clarke M, Sneade M, Yarnold JA, et al: International subarachnoid aneurysm trial (ISAT) of neurosurgical clipping versus endovascular coiling in 2143 patients with ruptured intracranial aneurysms: a randomised comparison of effects on survival, dependency, seizures, rebleeding, subgroups, and aneurysm occlusion. Lancet 366:809-817, 2005

16. Montaner J, Perea-Gainza M, Delgado P, Ribó M, Chacón $\mathrm{P}$, Rosell A, et al: Etiologic diagnosis of ischemic stroke subtypes with plasma biomarkers. Stroke 39:2280-2287, 2008

17. Park HK, Horowitz M, Jungreis C, Genevro J, Koebbe C, Levy E, et al: Periprocedural morbidity and mortality associated with endovascular treatment of intracranial aneurysms. AJNR Am J Neuroradiol 26:506-514, 2005

18. Pierot L, Cognard C, Anxionnat R, Ricolfi F: Ruptured intracranial aneurysms: factors affecting the rate and outcome of endovascular treatment complications in a series of 782 patients (CLARITY study). Radiology 256:916-923, 2010

19. Raymond J, Guilbert F, Weill A, Georganos SA, Juravsky L, Lambert A, et al: Long-term angiographic recurrences after selective endovascular treatment of aneurysms with detachable coils. Stroke 34:1398-1403, 2003

20. Righini M, Van Es J, Den Exter PL, Roy PM, Verschuren F, Ghuysen A, et al: Age-adjusted D-dimer cutoff levels to rule out pulmonary embolism: the ADJUST-PE study. JAMA 311:1117-1124, 2014

21. Shigematsu T, Fujinaka T, Yoshimine T, Imamura H, Ishii A, Sakai C, et al: Endovascular therapy for asymptomatic unruptured intracranial aneurysms: JR-NET and JR-NET2 findings. Stroke 44:2735-2742, 2013

22. Timp JF, Braekkan SK, Versteeg HH, Cannegieter SC: 
Epidemiology of cancer-associated venous thrombosis. Blood 122:1712-1723, 2013

23. Viñuela F, Duckwiler G, Mawad M: Guglielmi detachable coil embolization of acute intracranial aneurysm: perioperative anatomical and clinical outcome in 403 patients. J Neurosurg 86:475-482, 1997

24. von Elm E, Altman DG, Egger M, Pocock SJ, Gøtzsche PC, Vandenbroucke JP: The Strengthening the Reporting of Observational Studies in Epidemiology (STROBE) statement: guidelines for reporting observational studies. Lancet 370:1453-1457, 2007

25. Workman MJ, Cloft HJ, Tong FC, Dion JE, Jensen ME, Marx WF, et al: Thrombus formation at the neck of cerebral aneurysms during treatment with Guglielmi detachable coils. AJNR Am J Neuroradiol 23:1568-1576, 2002

26. Yamada NK, Cross DT III, Pilgram TK, Moran CJ, Derdeyn CP, Dacey RG Jr: Effect of antiplatelet therapy on thromboembolic complications of elective coil embolization of cerebral aneurysms. AJNR Am J Neuroradiol 28:17781782,2007

27. Yamane F, Ishihara S, Kohyama S, Kanazawa R, Ishihara H, Suzuki M, et al: Local thrombus formation at the coil-parent artery interface during endovascular coil embolization of cerebral aneurysms. J Neurol Surg A Cent Eur Neurosurg 73:358-368, 2012

\section{Disclosures}

The authors report no conflict of interest concerning the materials or methods used in this study or the findings specified in this paper.

\section{Author Contributions}

Conception and design: Fukuda. Acquisition of data: Handa, Koyanagi. Analysis and interpretation of data: Fukuda, Lo. Drafting the article: Fukuda, Lo. Critically revising the article: all authors. Reviewed submitted version of manuscript: all authors. Approved the final version of the manuscript on behalf of all authors: Fukuda. Statistical analysis: Fukuda. Administrative/technical/material support: Handa, Koyanagi. Study supervision: Yamagata.

\section{Correspondence}

Hitoshi Fukuda: Kurashiki Central Hospital, Kurashiki, Okayama, Japan.fukudaharpseal@gmail.com. 